\title{
Loss of Function Mutations in Filaggrin are Associated with Multiple Contact Positive Reactions
}

Peter Hull1,6, Denise Sasseville ${ }^{2}$, Melanie Pratt ${ }^{3}$, Youssef Elhaji ${ }^{1}$, Yuka Asai ${ }^{4}$, Mariam Abbas ${ }^{5}$ Deborah Michel ${ }^{6}$, Linda Campbell ${ }^{7}$, Irwin McLean ${ }^{7}$

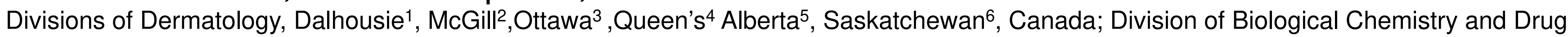
Discovery ${ }^{7}$, University of Dundee, UK

\section{Background}

Polysensitivity has been defined as the three or more positive patch tests. Patients with atopic dermatitis or other forms of eczema are more likely to develop polysensitivity. This suggests that defects in barrier function as caused by null mutations in FLG or other induced defects in barrier function may play a predisposing role. In this Canadian study we evaluated the association of loss of function mutations in FLG and polysensitivity.

\section{Methods}

Patients were ascertained over a 10 period from 3 University based patch test clinics at the Universities of Saskatchewan (PH), Ottawa (MP) and McGill University in Montreal (DS). Patients with 3 or more unrelated positive patch tests were included. In order to exclude skin barrier bypass, Nickel was excluded as were patients with metal implants, a prior history of hand dermatitis and stasis dermatitis with and without ulceration. The following prevalent European null mutations in FLG were sequenced, R501X, 2282del4, R2447X and S3247X. Sequencing of PCR products was performed by a core facility (DNA Sequencing and Services, University of Dundee, Dundee, United Kingdom) as well as at Dalhousie University. As prevalent mutations are not known for other ethnic groups in Canada, the study was confined to those with self identified European ancestry.

165 participants were included. In an additional 4 sequencing failed for all four mutations tested and they were excluded from the study. Single mutations failed in 2 cases (one each for R501X and S3247X).

\section{Control Population}

The control population were adult volunteers from Toronto, Ontario, whose DNA is commercially available. A total of 891 Canadian controls of Caucasian ethnicity were screened for the same FLG mutations. The presence or absence of coexisting atopic dermatitis is not known.

\section{Patient Demographics}

\begin{tabular}{|cccc|}
\hline & Combined & FLG Wild Type & FLG Null Mutations \\
\hline Female & $71 \%$ & $74 \%$ & $62 \%$ \\
\hline Average age & 52 & 54 & 46 \\
\hline AD & $34 \%$ & $32 \%$ & $46 \%$ \\
\hline Asthma & $22 \%$ & $20 \%$ & $35 \%$ \\
\hline Hay fever & $38 \%$ & $37 \%$ & $41 \%$ \\
\hline Food allergies & $18 \%$ & $16 \%$ & $29 \%$ \\
\hline FH AD & $37 \%$ & $34 \%$ & $29 \%$ \\
\hline FH Asthma & $33 \%$ & $33 \%$ & $53 \%$ \\
\hline FH Food Allergies & $17 \%$ & $16 \%$ & $21 \%$ \\
\hline Average no of Positives & 6 & 6 & 6 \\
\hline North American Series & 4.5 & 4.5 & 4.7 \\
\hline
\end{tabular}

\section{References}

1.de Jongh CM, Khrenova L, Verberk MM, Calkoen F, van Dijk FJ, Voss H, et al. Loss-of-function polymorphisms in the filaggrin gene are associated with an increased susceptibility to chronic irritant contact dermatitis: a case-control study. Br J Dermatol. 2008;159(3):621-7.

2. Carlsen BC, Andersen KE, Menne T, Johansen JD. Patients with multiple contact allergies: a review. Contact Dermatitis. 2008;58(1):1-8.

3. Carlsen BC, Johansen JD, Menne T, Meldgaard M, Szecsi PB, Stender S, et al Filaggrin null mutations and association with contact allergy and allergic contact dermatitis: results from a tertiary dermatology clinic. Contact Dermatitis 2010;63(2):89-95

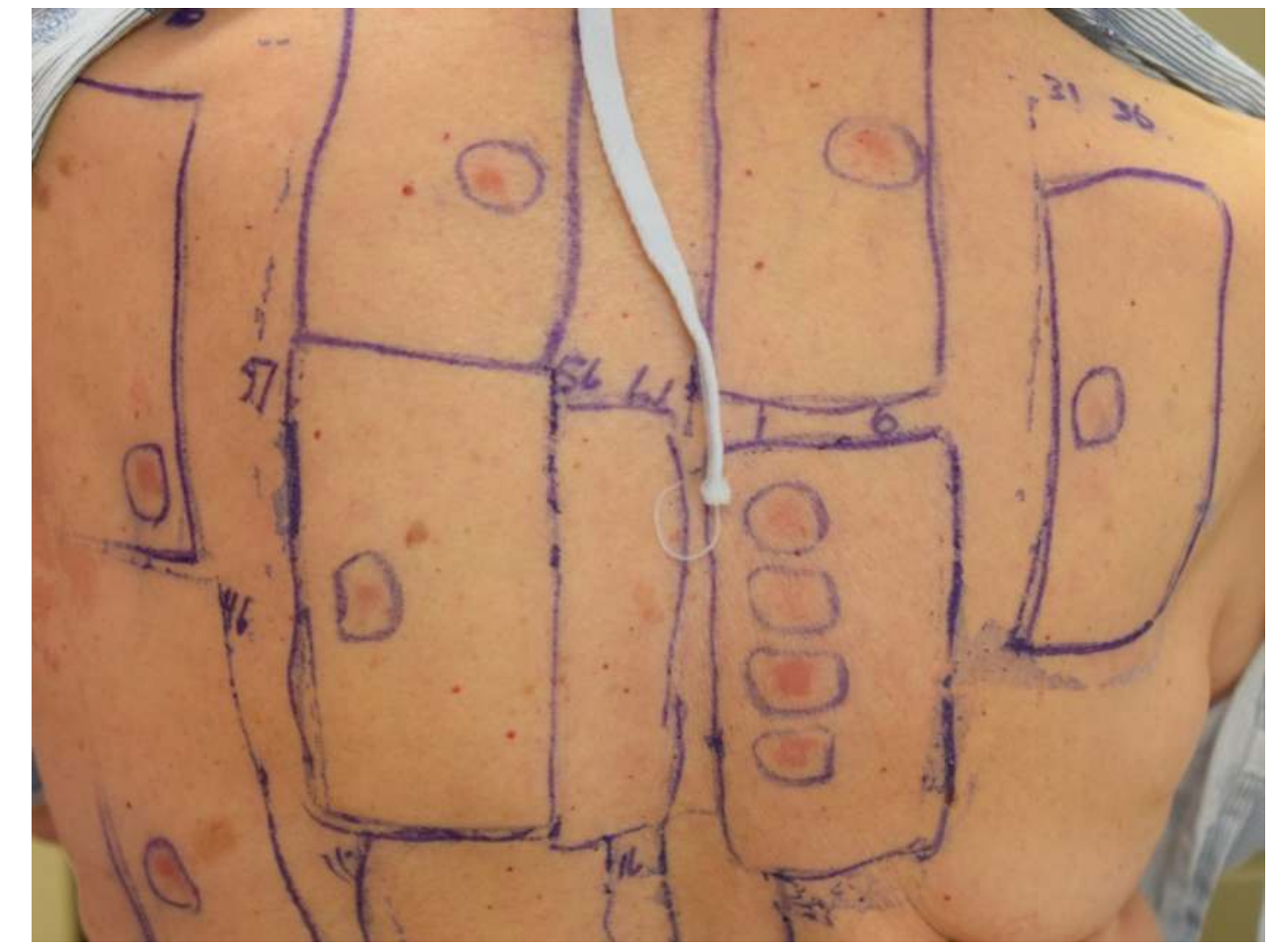

\section{Results}

Genotyping results and statistical analysis of FLG lossof-function mutations in 165 patients with polysensitivity and 891 Canadian controls

\begin{tabular}{|c|c|c|c|c|c|c|c|c|c|c|}
\hline & \multicolumn{2}{|c|}{$501 X$} & \multicolumn{2}{|c|}{ 2282del4 } & \multicolumn{2}{|c|}{ R2447X } & \multicolumn{2}{|c|}{ S3247X } & \multicolumn{2}{|c|}{ Combined } \\
\hline & Cases & Controls & Cases & Controls & Cases & Controls & Cases & Controls & Cases & Controls \\
\hline Wild Type & 134 & 859 & 135 & 846 & 136 & 883 & 136 & 884 & 137 & 793 \\
\hline $\begin{array}{l}\text { One FLG } \\
\text { Mutation }\end{array}$ & 13 & 34 & 9 & 46 & 2 & 11 & 3 & 11 & 24 & 9 \\
\hline $\begin{array}{l}\text { Compound } \\
\text { Het }\end{array}$ & & & & & & & & & 2 & 3 \\
\hline Homozygous & 1 & & 1 & & & & & 1 & 2 & 1 \\
\hline $\begin{array}{l}\text { Percentage } \\
\text { with } \\
\text { Mutations }\end{array}$ & $8.6 \%$ & $3.8 \%$ & $6.8 \%$ & $5.2 \%$ & $2.1 \%$ & $1.2 \%$ & $2.1 \%$ & $1.1 \%$ & $17 \%$ & $9.9 \%$ \\
\hline $\begin{array}{l}\text { Fisher's } \\
\text { Exact Test }\end{array}$ & \multicolumn{2}{|c|}{$P=0.005$} & \multicolumn{2}{|c|}{$P=0.426$} & \multicolumn{2}{|c|}{$P=0.698$} & \multicolumn{2}{|c|}{$P=0.4$} & \multicolumn{2}{|c|}{$P=0.036$} \\
\hline $\begin{array}{l}\text { Odds Ratio } \\
\text { (Cl) }\end{array}$ & \multicolumn{2}{|c|}{$\begin{array}{c}2.639596 \\
(1.2719 \text { to } 5.2030)\end{array}$} & \multicolumn{2}{|c|}{$\begin{array}{c}1.3623 \\
(0.5981 \text { to } 2.8190)\end{array}$} & \multicolumn{2}{|c|}{$\begin{array}{c}1.135027 \\
(0.1210 \text { to } 5.2843)\end{array}$} & \multicolumn{2}{|c|}{$\begin{array}{l}1.95 \\
(0.5725 \text { to } 6.6655)\end{array}$} & \multicolumn{2}{|c|}{$\begin{array}{c}1.6548 \\
\text { (1.0058 to } 2.6513)\end{array}$} \\
\hline $\begin{array}{l}\text { Z Statistic } \\
\text { Significance } \\
\text { level }\end{array}$ & \multicolumn{2}{|c|}{$\begin{array}{c}2.934 \\
P=0.0033\end{array}$} & \multicolumn{2}{|c|}{$\begin{array}{c}0.856 \\
P=0.3917\end{array}$} & \multicolumn{2}{|c|}{$\begin{array}{c}0.858 \\
P=0.3912\end{array}$} & \multicolumn{2}{|c|}{$\begin{array}{c}1.005 \\
P=0.3159\end{array}$} & \multicolumn{2}{|c|}{$\begin{array}{c}2.155 \\
P=0.0311\end{array}$} \\
\hline
\end{tabular}

\section{Top 10 Positive Allergens}

\begin{tabular}{|c|c|c|}
\hline Combined & FLG Wild Type & FLG Null Mutations \\
\hline Fragrance mix 1 & Fragrance mix 1 & Formaldehyde \\
\hline Formaldehyde & Formaldehyde & Balsam of Peru \\
\hline Quaternium 15 & Quaternium 15 & Cobalt \\
\hline Balsam of Peru & Balsam of Peru & Chromate* $^{*}$ \\
\hline Nickel & Bacitracin & Quaternium $15^{*}$ \\
\hline Bacitracin* & Nickel & Lanolin* \\
\hline Cobalt $^{*}$ & Colophony & Fragrance Mix $1^{*}$ \\
\hline Colophony $^{* *}$ & Cobalt ${ }^{*}$ & Neomycin \\
\hline Neomycin** & Neomycin* & Colophony** \\
\hline Lanolin & PPD & Nickel** \\
\hline PPD & Lanolin & Propylene glycol** \\
\hline
\end{tabular}

\section{Discussion.}

Loss of function mutations are a major predisposing factor in atopic dermatitis. The role in allergic contact dermatitis is less clear. Loss of function mutations in FLG have been associated with irritant dermatitis, which in turn would damage barrier function and predispose to an allergic contact dermatitis. ${ }^{1}$ Polysensitivity has been defined as the finding of 3 or more positive patch tests. ${ }^{2}$ In a previous study, polysensitivity was not associated with FLG mutations. ${ }^{3}$ In this study, we examined this relationship, but excluded situations where the barrier may have been either by-passed or damaged.

\section{Conclusion}

In this Canadian group of patients, polysensitivity was significantly associated with loss of function mutations in FLG. 